\title{
SwissDRG: stratégie pour le développement futur
}

\section{Beatrix Meyer}

Responsable du département Tarifs et économie de la santé pour les médecins hospitaliers
Correspondance: FMH

Froburgstrasse 15 CH-4600 Olten Tél. 0313591111 Fax 0313591112
Depuis plus d'une année, les hôpitaux de soins aigus facturent selon le système de forfaits par cas SwissDRG. Il est incontesté que la structure tarifaire SwissDRG doit poursuivre son développement et s'améliorer. Au cours d'âpres négociations, les partenaires de SwissDRG SA ont déterminé dans quelle direction le système SwissDRG devra évoluer à l'avenir. Lors de ces négociations, la FMH s'est appuyée sur sa prise de position [1] ainsi que sur les votes et décisions de divers organes, à savoir le comité SwissDRG et le groupe de travail SwissDRG de la FMH, le Comité central et l'Assemblée des délégués de la FMH. Ciaprès, nous présentons un aperçu de la façon dont les demandes du corps médical ont été prises en compte concrètement dans le document stratégique [2] de SwissDRG SA.

\section{Une structure tarifaire différenciée comme objectif stratégique}

Pour équilibrer cette structure tarifaire encore inadéquate, il est nécessaire de recourir de manière transitoire à des taux de base (baserates) plus élevés pour les hôpitaux universitaires et ceux qui offrent une médecine hautement spécialisée. Toutefois, du point de vue de la FMH, cette mesure d'urgence ne saurait devenir une solution durable, sinon elle menacerait la compétitivité de ces hôpitaux. Une prise en charge des lacunes financières par les cantons et finalement par les contribuables ne correspond pas non plus à l'esprit du nouveau financement hospitalier. L'année dernière a montré à quel point les négociations tarifaires étaient difficiles. La fausse conception du Surveillant des prix selon laquelle la structure tarifaire tiendrait suffisamment compte des prestations n'y est pas étrangère. Si la Suisse entend atteindre une rémunération conforme à la prestation, alors l'amélioration du système tarifaire SwissDRG est la seule voie! La FMH salue donc l'objectif stratégique de SwissDRG SA qui est de créer une structure tarifaire différenciée. Par ailleurs, SwissDRG SA a tenu compte des recommandations de la FMH demandant de considérer les développements du système allemand de forfaits par cas G-DRG et de suivre étroitement les nouveaux hôpitaux du réseau afin d'améliorer la qualité des données hospitalières. En outre, dès 2015, il s'agira d'inclure dans la structure tarifaire, selon des critères transparents, les frais d'utilisation des infrastructures. Ainsi, nous en aurons enfin terminé avec cette tragédie autour de la fixation du supplément normatif sur le prix de base pour la rémunération des frais d'utilisation des infrastructures. Entre-temps, la FMH, alliée à d'autres partenaires, a pu éviter que le Conseil fédéral ne prescrive un supplément normatif de seulement 9\% pour 2013 [3].

\section{8 rétributions additionnelles pour la version 3.0 de SwissDRG}

SwissDRG SA part du principe qu'un système tarifaire comprenant près de 1200 forfaits par cas, rétributions additionnelles comprises, tient parfaitement compte des différences de frais entre fournisseurs de prestations [4]. Les rétributions additionnelles sont indispensables pour une rémunération adéquate des médicaments, produits sanguins, implants et méthodes au coût élevé, s'étendant sur plusieurs DRG. Elles permettent d'éviter que le nombre de forfaits par cas augmente de manière inutile par une scission des DRG existants. Après de nombreuses interventions de la FMH, SwissDRG SA a tout de même calculé 28 rétributions additionnelles [5] pour la version 3.0 de SwissDRG. En comparaison avec la version 2.0, qui prévoyait cinq rétributions additionnelles, cela représente un pas important dans la bonne direction. La demande de la FMH visant à reprendre sous forme helvétisée, en cas d'insuffisance des données suisses, les rétributions additionnelles allemandes qui avaient été proposées par les sociétés de discipline médicale suisses n'a pas obtenu la majorité. SwissDRG SA souhaite calculer les rétributions additionnelles uniquement sur la base des données hospitalières suisses et ne reprendre que celles qui représentent au moins CHF 50000.- au budget d'un hôpital. Un hôpital suisse en particulier a fait, l'année dernière, un effort considérable et a fourni à SwissDRG SA des données pour la calculation des rétributions additionnelles. Pour les relevés en cours, tous les hôpitaux sont priés de fournir des données d'un haut niveau de qualité, afin que d'autres rétributions additionnelles proposées puissent être concrétisées dans les futures versions.

\section{Procédure de propositions accélérée pour les innovations}

Les expériences réalisées jusque-là avec le processus d'indemnisation des méthodes innovatrices en matière d'investigation et de traitement ont montré que les réserves de la FMH sont justifiées. Le processus [6] est bien trop compliqué et extrêmement fastidieux. Par exemple, l'intervalle entre la proposition d'un code analogique et la publication de celui-ci sur le site internet de SwissDRG a duré dix mois. C'est pourquoi la FMH demande depuis longtemps que le processus soit révisé, afin que les innovations soient indemnisées rapidement et en conformité avec les prestations. SwissDRG SA va maintenant proposer un processus accéléré pour la procédure de propositions en vue de l'indemnisation des innovations coûtant au moins CHF 50 000.-par institution. 


\section{Valeurs-seuils irréalistes pour les cas extrêmement coûteux}

Il existe des cas si complexes et si coûteux que les dépenses qu'ils engendrent sont très loin d'être couvertes. Lors de la dernière rencontre organisée par le domaine «Tarifs et économie de la santé pour les médecins hospitaliers» de la $\mathrm{FMH}$, les présidents ainsi que les délégués DRG des sociétés de discipline médicale et des associations faîtières ont discuté intensivement des problèmes liés à la sous-indemnisation des cas de traitement coûteux et complexes. Un article complet à ce sujet paraîtra prochainement dans le Bulletin des médecins suisses. SwissDRG SA a certes pris note des demandes du corps médical, mais a recours à des valeurs-seuils irréalistes. Dans son document stratégique, SwissDRG affirme que «des solutions pour rémunérer les cas très coûteux (par ex. à partir de CHF 500000.-) avec un risque de coûts unilatéral pour les hôpitaux doivent le cas échéant être élaborées hors structure tarifaire si le système de forfaits par cas ne permet pas, de manière prouvée, d'établir une différenciation (écart avec les coûts moyens > CHF 50000.-)». Selon la FMH, la valeur de CHF 50000.- est fixée trop haut et l'exemple des CHF 500000.- induit en erreur. La FMH compte intervenir pour que cet exemple irréaliste de $\mathrm{CHF}$ 500000.- ne soit pas repris comme critère, sinon la recherche d'une solution pour le financement adéquat des cas très coûteux tournerait à la farce.

\section{Structures tarifaires séparées pour la réadap- tation, la psychiatrie et les soins palliatifs}

S'agissant des structures tarifaires développées spécifiquement pour la réadaptation et la psychiatrie, SwissDRG SA a fixé le délai d'introduction à 2015/2016. Pour les soins palliatifs, SwissDRG SA vérifie actuellement s'il convient également d'élaborer une structure tarifaire séparée. La FMH continue de faire son possible pour que les demandes du corps médical soient prises en compte lors du développement de ces nouvelles structures tarifaires hospitalières. La prise de position de la FMH sur le développement de la structure tarifaire ST Reha [7] a suscité un débat très positif et constructif chez les partenaires. Au sein du comité de pilotage TARPSY siège désormais un deuxième observateur du corps médical, afin qu'il soit explicitement tenu compte du point de vue de la psychiatrie d'enfants et d'adolescents [8].

\section{Facteur temps déterminant}

Comme la FMH l'avait depuis longtemps demandé, SwissDRG SA s'est fixé pour objectif d'accélérer le développement de SwissDRG. Ainsi, à l'avenir, le développement de la nouvelle version de SwissDRG se fondera sur les données de l'année précédente. Le but est donc de raccourcir d'un an le cycle de développement. Contrairement aux avis de certains partenaires, il a été décidé, dans le sens de la FMH, que la procédure de propositions en vue de l'amélioration du système SwissDRG aurait également lieu annuellement à l'avenir.
Même si aucune précision de temps n'est indiquée dans la stratégie, il est clair qu'il faut prendre sans retard les mesures les plus importantes pour atteindre les objectifs. En priorité, il faut d'une part que SwissDRG SA suive étroitement la saisie des données hospitalières qui vient de débuter (relevé des données normales y compris relevé détaillé pour les médicaments, produits sanguins, implants et méthodes au coût élevé) pour la version 4.0 de SwissDRG. D'autre part, il est important que les travaux en vue de définir un processus pour l'indemnisation des innovations et des cas aux coûts élevés soient très vite mis en route, avec la participation des divers partenaires.

\section{Références}

1 cf. Prise de position de la FMH sur la version 2.0 de SwissDRG et recommandations pour le développement futur du système, du 21.05.2012, www.fmh.ch $\rightarrow$ Tarifs $\rightarrow$ SwissDRG $\rightarrow$ Informations de fond.

2 cf. www.swissdrg.org

3 cf. www.fmh.ch $\rightarrow$ Politik \& Medien $\rightarrow$ Vernehmlassungen $\rightarrow$ Stellungnahme der FMH zum Entwurf der Teilrevision der KVV-Verordnung und zur Abgeltung der Anlagenutzungskosten (seulement en allemand).

4 La version 2.0 de SwissDRG contient 991 forfaits par cas.

5 Pour la version 3.0 de SwissDRG, de nouvelles rétributions additionnelles tarifées ont été calculées pour les cytostatiques (Pemetrexed, Rituximab, Trastuzumab, Cetuximab, Bevazizumab, Bortezomib), les immoglobulines humaines (polyvalentes, contre les antigènes de surface de l'hépatite $\mathrm{B}$, cytomégalovirus), les antimycotiques (Caspofungine, Amphothéricine B liposomale, Voriconazol, Posaconazol, Anidulafungine), les anticorps (Infliximab), les concentrés d'érythrocytes et de plaquettes (CP normal, CP d'aphérèse et CP liées au patient), les stents auto-expansifs dans le tractus gastro-intestinal, l'implantation sélective de spirales métalliques (coils), l'aphérèse thérapeutique. Les rétributions additionnelles qui existaient déjà dans la version 2.0 de SwissDRG ont été adaptées voire élargies pour la version 3.0. Ainsi l'indication pour la rétribution additionnelle non tarifée «cœur artificiel» a été élargie de «bridge to transplant» à «destination therapy». La rétribution additionnelle non tarifée pour les facteurs de coagulation chez les hémophiles a été élargie pour l'indication «maladie du système circulatoire acquise durablement» selon la liste de diagnostics donnée. En outre, trois autres facteurs ont été complétés (facteurs VII plasmatique et facteur XIII, et combinaison du facteur de von Willebrand avec le facteur VIII). Deux des trois rétributions additionnelles pour suppléance rénale ont été nouvellement tarifées pour la version 3.0 sur la base de chiffres suisses, l'une a été reprise de la version 2.0 de SwissDRG sans changement. Demeure réservée l'approbation de la version 3.0 de SwissDRG par le conseil d'administration de SwissDRG SA et par le Conseil fédéral.

6 cf. Schéma de la FMH et de H+ sur le financement de prestations et méthodes innovatrices, www.fmh.ch $\rightarrow$ Tarifs $\rightarrow$ SwissDRG $\rightarrow$ Procédure de propositions, voir Documents à joindre à la demande de proposition.

7 cf. Prise de position de la FMH sur la structure tarifaire suisse pour la réadaptation (ST Reha), www.fmh.ch $\rightarrow$ Tarifs $\rightarrow$ Autres tarifs hospitaliers $\rightarrow$ Réadaptation ST Reha.

8 Le Dr Matthias Hilpert et désormais aussi le Dr Jürg Unger représentent le corps médical dans le comité de pilotage TARPSY en qualité d'observateurs. Pour un résumé du projet TARPSY, voir: Hilpert M. TARPSY - un nouveau système tarifaire pour la psychiatrie hospitalière. Bull Méd Suisses. 2012;93(49):1811-2. www.fmh.ch $\rightarrow$ Tarifs $\rightarrow$ Autres tarifs hospitaliers $\rightarrow$ Psychiatrie TARPSY 